\title{
Retraction Note to: Local Feature Weighting for Data Classification
}

\author{
Gengyun $\mathrm{Jia}^{2}$, Haiying Zhao ${ }^{1,2(\bowtie)}$, Zhigeng $\operatorname{Pan}^{3}$, \\ and Liangliang Wang ${ }^{4}$ \\ ${ }^{1}$ Mobile Media and Cultural Computing Key Laboratory of Beijing, \\ Century College, BUPT, White Bear Lake, China \\ zhy.yn@163.com \\ ${ }^{2}$ School of Information and Communication Engineering, \\ Beijing University of Posts and Telecommunications, Beijing, China \\ ${ }^{3}$ Digital Media and HCI Research Center, \\ Hangzhou Normal University, Hangzhou, China \\ ${ }^{4}$ Xinjiang Teacher's College, Xinjiang, China
}

\section{Retraction Note to: \\ Chapter "Local Feature Weighting for Data Classification" in: \\ Z. Pan et al. (Eds.): \\ Transactions on Edutainment XIII, LNCS, \\ DOI: 10.1007/978-3-662-54395-5_25}

The paper starting on page 293 of this volume has been retracted because a significant portion of the work was copied from the paper "Local Feature Selection for Data Classification" by Narges Armanfard, James P. Reilly, and Majid Komeili, published in 2016 in the IEEE TRANSACTIONS ON PATTERN ANALYSIS AND MACHINE INTELLIGENCE, VOL. 38. 